\title{
Analyse géopolitique des enjeux d'une politique de puissance: le cas de la science et de l'innovation en Russie
}

\author{
Kevin Limonier ${ }^{1}$
}

Depuis 2009, c'est à grand renfort médiatique et financier que le gouvernement russe promeut le développement du technoparc de Skolkovo. Identifié dans la presse par de prestigieuses épithètes telles que «Silicon Valley russe», «Smart City» ou encore «Innograd» (ville d'innovation), le projet monumental du président Medvedev devrait bénéficier d'au moins 54 milliards de roubles (1,35 milliard d'euros) de subventions fédérales d'ici 2013, alors qu'une deuxième tranche encore plus importante est prévue à l'horizon 2014². L'objectif est de créer dans la banlieue de Moscou une ville entièrement nouvelle entièrement dédiée à la recherche et à l'innovation, et bénéficiant des plus récentes commodités technologiques. Skolkovo se veut ainsi le symbole d'une modernisation sans précédent de l'économie russe, afin de sortir celle-ci de l'ornière de la rente énergétique. ㄱ. Tablant sur son potentiel intellectuel et scientifique, la Russie entend provoquer à Skolkovo un afflux massif d'investissements étrangers afin de donner au pays l'impulsion nécessaire au développement d'une économie de la connaissance selon les canons occidentaux du «développement durable». Si d'importants contrats ont déjà été signés avec de grandes entreprises étrangères telles que Alstom, Schneider, Apple, Boeing ou encore Microsoft, la ville intelligente du futur demeure pour l'instant à l'état de projet, et les travaux d'aménagement viennent à peine de commencer et devraient se poursuivre jusqu'en 2014, date à

1. Doctorant de l'Institut français de géopolitique, université Paris-VIII.

2. Kommersant', édition du 5 août 2010. 


\section{HÉRODOTE}

laquelle un sommet du G20 devrait y être organisé en guise d'inauguration. Les grandes firmes internationales, attirées par les subventions et la perspective d'une délocalisation d'une partie de leur branche $\mathrm{R} \& \mathrm{D}$ dans un pays où les coûts marginaux seraient moindres qu'en Europe ou qu'en Amérique du Nord, regardent pour l'instant le projet avec confiance.

À l'étranger, le projet Skolkovo suscite également intérêt et optimisme d'une grande partie de la presse et des experts en économie. Par exemple, en octobre 2011, le magazine The Atlantic consacrait à la «Silicon Valley russe» un long article élogieux, intitulé «The next Russian revolution» («La prochaine révolution russe »). De nombreuses études occidentales d'intelligence économique en font également le symbole d'une probable renaissance fondée sur la mise en valeur d'un patrimoine matériel, technique et humain latent dans l'économie russe depuis la chute de l'URSS $^{3}$. Skolkovo serait ainsi la vitrine mondiale d'une idée russe lancée à la face du monde et allègrement reprise par les médias : le renouveau.

Souvent interchangeable avec d'autres termes du même champ lexical tels que «réaffirmation», le «renouveau » établit en effet un lien intrinsèque entre la nouvelle puissance russe et des formes antérieures de puissance, au premier lieu desquelles se situe celle de l'Union soviétique.

Skolkovo pourrait à première vue s'apparenter à une vitrine de cette politique de renouveau, de mise en avant des domaines historiques d'excellence de la Russie en termes de science et d'innovation. Si le projet de Medvedev se veut d'abord le symbole d'une modernisation de l'économie nationale qui passerait par une mutation profonde des mécanismes russes traditionnels de la science et de la recherche, il n'en demeure pas moins que celui-ci s'appuie sur un savoir-faire, des traditions et même une représentation de la science fortement imprégnés par l'expérience soviétique. La «ville intelligente» sera d'ailleurs organisée en cinq secteurs qui correspondent aux domaines historiques d'excellence de la Russie en matière de hautes technologies (espace, biochimie, nucléaire, informatique et énergie). Sur la page Internet du cluster espace, on peut par exemple lire en phrase d'accroche que «les activités spatiales constituent une partie intégrante de l'héritage scientifique, technique et spirituel de la Russie».

Cet article propose ainsi d'interroger, avec la méthode géopolitique, les processus de formation d'un discours du renouveau fondé sur la mise en scène d'un patrimoine matériel, technique et humain issu de l'époque soviétique. D'apparence linéaire et pilotée par le pouvoir central, ce processus est au contraire

3. Lire à ce titre: De Jong P. (dir.), Les Ventes d'armes au service du renouveau de la Russie, étude de l'École de guerre économique (EGE), février 2012.

194 
le résultat de rivalités entre divers réseaux de pouvoir agissant à des niveaux géographiques et politiques très variés.

Au-delà de l'exemple de Skolkovo - qui, d'ailleurs, présente des particularités qui seront explicitées plus loin -, le pouvoir russe a en effet construit depuis le début des années 2000 un discours d'aspiration à la puissance qui entend s'imposer comme qualificatif général du pays sur la scène internationale et dans l'opinion publique mondiale. La mise en scène d'un patrimoine issu de l'époque soviétique, loin de se borner au domaine scientifique, est une stratégie de communication politique qui a investi nombre de secteurs d'activité symboliques de la puissance de l'État, et dont le reflet général procède de l'emboîtement d'enjeux identitaires et politiques à différents niveaux géographiques. Héritée du système socialiste de planification centralisée, la répartition fonctionnelle des forces militaires, industrielles et économiques sur le territoire postsoviétique met à la disposition du géographe un certain nombre de territoires précis dont la fonction ordonne l'identité.

L'étude, au niveau local, des interactions entre l'infrastructure de puissance et la réalité urbaine permet alors de mettre en lumière un mécanisme d'identification mutuelle qui constitue la base d'une analyse géopolitique des représentations de la puissance russe au niveau régional, national ou mondial, ainsi que les rivalités qui en découlent. Certaines villes monofonctionnelles de taille moyenne peuvent alors devenir de véritables laboratoires pour l'analyse géopolitique, permettant dans leur étude détaillée la compréhension de certains mécanismes et rivalités qui conditionnent une politique de puissance au niveau global ${ }^{4}$.

Dans le cas de la science et de l'innovation, la mise en scène du renouveau d'infrastructures datant de l'époque soviétique peut ainsi être analysée grâce à une démarche similaire. L'organisation territoriale de la science russe, héritée de

4. Une ville comme Sébastopol (dont l'histoire commence bien avant l'URSS) est ainsi d'abord représentée par sa flotte dans une interaction historique et identitaire complexe qui a des répercussions jusqu'au niveau mondial, le maintien dans ce port ukrainien d'une force militaire russe ne répondant pas seulement à des impératifs purement militaires ou stratégiques. Les 4 milliards de dollars actuellement déboursés par la Russie chaque année pour y maintenir les neuf navires militaires vieillissants qui composent la flotte de mer Noire s'expliquent surtout dans une démarche politique qui tient à la nature de la ville. Fondée par et pour des marins en 1783, Sébastopol constitue le dernier avant-poste de l'empire sur les mers chaudes. Son histoire, en résonant de grandes batailles et de faits héroïques au service de l'empire et de l'URSS, fait aujourd'hui de la ville un symbole d'une histoire commune; le maintien d'une force militaire russe y perpétue l'idée d'une communauté identitaire et mémorielle qui dépasserait les cultures nationales issues de la chute de l'Union soviétique. L'analyse locale des mobilisations politiques et des interactions entre ville et flotte permet de mieux comprendre certains enjeux régionaux, tels que la rivalité linguistique en Ukraine [Limonier, 2010]. 


\section{HÉRODOTE}

l'URSS, demeure en effet principalement organisée sur ce modèle fonctionnel évoqué plus haut. Il en résulte l'existence d'un véritable «archipel scientifique» russe, composé de villes dédiées à la recherche. Cet archipel, en cela qu'il concentre une majeure partie des capacités de recherche et d'innovation du pays, constitue un ensemble de «laboratoires géopolitiques» dont l'étude devrait permettre, par l'appréciation d'enjeux et de rivalités locaux, de mieux saisir certaines tendances générales propres à la Russie dans le cadre de sa politique de puissance et de renouveau scientifique. Il convient d'ailleurs de dire ici que, à l'exception notable du cosmodrome de Baïkonour, les lieux de la mise en scène du patrimoine scientifique de l'URSS se situent à l'intérieur des frontières de la Fédération de Russie. Les enjeux et rivalités inhérents à ces territoires et à leur renouveau concernent alors divers niveaux et réseaux de pouvoir allant du fédéral au local.

\section{Éléments de contextualisation}

Cependant, une telle étude nécessite d'abord une mise en contexte concernant la nature générale des discours appliqués au renouveau scientifique russe depuis l'arrivée au pouvoir de Vladimir Poutine jusqu'aux récentes contestations citoyennes nées dans l'hiver 2011. Celles-ci, n'épargnant pas le discours officiel sur la science et l'innovation, placent dans le débat public des rivalités de pouvoir latentes depuis deux décennies, et qui constituent l'être profond d'une politique de renouveau en réalité fondée sur la négociation et l'entente entre plusieurs réseaux d'influence.

\section{La science, composante d'une représentation de la Russie comme puissance}

en renouveau

La science constitue l'un des grands héritages russes de la puissance soviétique. Cependant, la mise en scène de son renouveau se distingue, par sa nature économique, de celle d'autres structures concentrant un fort potentiel symbolique (comme l'armée par exemple).

Si l'on s'en tient par exemple à la puissance militaire, la construction d'une frégate sera ainsi forcément déficitaire d'un point de vue strictement économique. En revanche, la puissance scientifique en économie de marché se détermine également par ses capacités de financement et de capitalisation de la connaissance, voire de rentabilisation dans le cadre d'un passage de la recherche fondamentale à l'innovation, si un champ de recherche répond par exemple à un besoin sur le 
marché. Or le domaine scientifique russe s'est retrouvé dans la quasi-impossibilité de réformer son système de financement sur des bases privées après le désengagement massif de l'État au début des années 1990, exception faite du secteur aérospatial. Celui-ci a bénéficié des conditions favorables d'un marché oligopolistique sur lequel il est vite devenu performant, notamment grâce à d'importants investissements étrangers et à une diversification des activités (lancements commerciaux, tourisme spatial...).

De même, si quelques spin-off 5 à succès ont pu voir le jour dans les années qui ont suivi la chute de l'URSS, elles demeurent tout à fait marginales alors que la perte de confiance en l'appareil russe de science et d'innovation n'incite ni les consommateurs à privilégier des produits de haute technologie conçus en Russie, ni les étudiants à s'engager dans une carrière scientifique. Le défi qui s'est posé au pouvoir dès le début des années 2000 , dans une perspective de recentralisation de la décision, a donc été de moderniser les structures économiques et politiques de la recherche scientifique, tout en favorisant l'émergence d'une «économie de la connaissance» fondée sur l'innovation. La rente énergétique russe demeure en effet un danger pour la santé économique du pays (lorsque l'unité de gaz tombe en dessous de 120 dollars, le budget fédéral n'est plus à l'équilibre), alors que la Russie dispose encore d'un large patrimoine scientifique pouvant mener à un développement économique durable par l'innovation et la recherche.

C'est ainsi que, dès 2003, le secteur scientifique et technologique russe est identifié par le pouvoir comme «intérêt vital de la Fédération». Au-delà de la signification économique du terme, il s'agissait pour Vladimir Poutine de procéder à une mise en avant massive des symboles de la puissance, et dont la portée patriotique était largement assumée. D'ailleurs, toute la communication du Président dans la décennie 2000 était fondée sur ce principe, à grand renfort médiatique. La doctrine de la «résolution au cas par cas» des grands maux du pays a ainsi donné lieu à de nombreuses mises en scène, parfois spectaculaires, pour répondre aux attentes d'une population désorientée par une décennie de crise économique et morale profonde. L'arrestation de Mikhaïl Khodorkovski dans le cadre de la politique de «recentralisation du pouvoir» en est un exemple évocateur. En termes économiques, la constitution de vastes consortiums d'industries de pointe s'est également faite dans le strict respect de la «verticale du pouvoir», lorsque

5. Une spin-off est une PME créée par d'anciens membres d'un institut de recherche, d'une université ou d'un laboratoire dans l'objectif de développer une activité commerciale sur la base de leur savoir-faire scientifique et technique. En Russie, ce processus a pu être favorisé dans les années 1990 par la chute des budgets alloués à la recherche scientifique (qui obligeait de nombreux chercheurs à trouver une nouvelle source de revenus), comme par la «soif d'entreprendre» de certains chercheurs. 


\section{HÉRODOTE}

par exemple tous les constructeurs aéronautiques russes sont réunis, par le fait du prince, dans une même organisation dont l'État est l'actionnaire majoritaire (OAK).

La popularité du «poutinisme» dans la première moitié des années 2000 fut en grande partie fondée sur la perception positive de telles politiques par l'opinion publique, qui a pu y voir le signe d'un rétablissement de la Russie dans son statut de grande puissance. Un intéressant sondage mené à Vladimir en 2004 s'est penché sur la nature de cette idée russe de la puissance. À la question «Qu'est-ce qui fait d'un État une grande puissance (derjava)?», $43 \%$ des sondés ont répondu «un haut niveau de vie » et «une économie développée». En dernière position (10\%) arrivaient «les droits et libertés démocratiques », derrière «un passé héroïque et glorieux » ou «une armée puissante» [Petrosian, Svincov, 2008]. La préoccupation de ce panel représentatif était alors centrée sur les conditions de vie économiques comme force structurante du discours de renouveau de la puissance russe, ainsi que sur le rapport au passé et à l'identité. C'est bien en s'appuyant sur une aspiration quasi nostalgique à la stabilité économique et politique que le pouvoir a fondé sa communication politique, à travers la mise en scène de la renaissance des secteurs qui faisaient jadis la gloire du pays. En partant du célèbre postulat poutinien que la chute de l'URSS constitue «la plus grande catastrophe du XX $\mathrm{X}^{\mathrm{e}}$ siècle», le pouvoir se devait d'inventer ses propres codes, sa propre esthétique dans la mise en scène de cette stabilité retrouvée.

Le terme de derjavnost' a ainsi fait son apparition dans la littérature politique et philosophique à partir de la toute fin des années 1990, pour définir la nature profonde de cette nouvelle tendance que certains appelaient de leurs vœux avant même l'arrivée au pouvoir de Vladimir Poutine. Intraduisible en français, il est cependant possible d'expliciter le sens du mot par l' «aspiration d'un pays à devenir une grande puissance ». Au-delà de cette définition que donne le dictionnaire, de nombreux politistes, philosophes se sont attachés dans la première moitié des années 2000 à théoriser cette aspiration à la grandeur et à la puissance en accord avec les héritages profonds de la Russie. Les définitions variaient alors de l'alliance poétique avec le sobornost' («communauté des croyants ») et l'obshinost' («génie social russe») [Bol'shakov, Ermackov, 1999] à «l'indépendance de l'État, motivée une politique, ayant une influence significative sur les affaires internationales ». Selon Ju. M. Baskakova, la derjava («grande puissance ») nourrie par l'idée de derjavnost' se distinguerait par une série caractéristique allant de «la capacité d'un État à résoudre les problèmes internationaux en accord avec ses principes de souveraineté », à « la force de dissuasion nucléaire » [Baskakova, 2008]. 
ANALYSE GÉOPOLITIQUE DES ENJEUX D’UNE POLITIQUE DE PUISSANCE

"Clientélisme, fric et copinage », une nouvelle représentation du renouveau depuis décembre 2011?

Dans le cas de la science et de l'innovation, ce principe de puissance souveraine a amené les dirigeants russes à imaginer de nouvelles politiques de promotion d'un secteur qui, au-delà de ses capacités économiques, constitue une partie intégrante du patrimoine identitaire russe.

Le projet de Skolkovo est lui-même imprégné de cette tendance, bien qu'il soit la création de Dmitri Medvedev, dans une esthétique qui détonne avec les canons du poutinisme: utilisation massive des réseaux sociaux, valorisation du jeune entrepreneur investi dans l'économie numérique... Les images renvoyées par Skolkovo et la commission pour la modernisation du pays (appelée de manière très évocatrice «i-Russia») sont le fruit de réseaux de pouvoir sensiblement différents de ceux avec lesquels Vladimir Poutine avait l'habitude de traiter. La montée en puissance de Dmitri Medvedev a ainsi induit une première rupture dans le style et le choix politique des stratégies de mise en scène du renouveau, alors qu'une seconde rupture, bien plus profonde, est marquée par la contestation grandissante depuis décembre 2011. D'ailleurs, l'identification de Skolkovo par cette contestation à travers l'humour, la raillerie et le cynisme est tout à fait évocatrice de nouvelles perceptions qui émergent au sein de la population quant à l'identification de ce renouveau comme représentation du pays et de sa puissance.

Des bons mots aux comparaisons amusantes, les figures russes de l'opposition adorent critiquer en ligne le grand projet de Dmitri Medvedev. C'est d'ailleurs devenu l'un des sujets qui fédèrent cette dissidence numérique dont on retrouve beaucoup de membres lors des manifestations et «promenades» actuellement régulières à Moscou. Skolkovo serait selon eux une «nouvelle Roubliovka» (le quartier au sud-est de Moscou où résident les oligarques et milliardaires proches du pouvoir), un «village Potemkine» destiné à enrichir quelques privilégiés. Force est de constater que le rôle central dévolu par le pouvoir dans ce projet à des milliardaires notoires (Vekselberg notamment) n'incite pas les opposants à la confiance. De même, la holding d'Etat Rosnanotech, fondée afin de favoriser l'émergence des nanotechnologies dans les différentes branches de la recherche et de l'innovation et très impliquée dans le projet Skolkovo, est la cible des railleries du Web. Son directeur, Anatoli Tchoubaïs, est un milliardaire controversé et assez impopulaire qui fut l'un des artisans des réformes économiques des années 1990.

L'intérêt de la contestation autour de Skolkovo pour ce travail réside alors dans le fait que ce mouvement se fonde sur la dénonciation de certaines logiques clientélistes et rivalités de pouvoir qui, en exaspérant la société civile naissante, justifient la pertinence d'un travail géopolitique multiscalaire sur les mécanismes de mise en place d'une représentation officielle du renouveau scientifique et des 


\section{HÉRODOTE}

jeux de réseaux qui en résultent depuis le début des années 2000. Il existe en effet depuis cette période un décalage grandissant entre, d'une part, les représentations du renouveau voulues par les élites et, d'autre part, des attentes démocratiques un temps contentées par les énormes bénéfices dégagés par la rente énergétique, qui menèrent à la modernisation des infrastructures comme au retour de l'Etat dans les financements de la recherche. Ici, l'analyse des lieux de la réalisation du renouveau et de sa mise en scène s'impose pour comprendre la rupture qui mène aujourd'hui à sa critique, au-delà de l'image générale que le pays entend renvoyer à travers le terme de «renouveau». Les mécanismes de cette représentation sont alors à trouver dans le «laboratoire géopolitique» que constitue l'archipel scientifique russe.

\section{De l'archipel scientifique soviétique aux naukograds de la Fédération de Russie: l'étude d'une représentation territoriale inventée à des fins politiques}

L'archipel scientifique de l'URSS : un laboratoire géopolitique

Cette carte présente l'organisation territoriale de la recherche et de l'innovation au lendemain de la chute de l'URSS, et doit être interprétée suivant trois points majeurs.

Premièrement, ce territoire est pensé de manière fonctionnelle dans un contexte de planification centralisée. Le complexe scientifique de l'URSS était alors organisé en archipel, avec de très fortes interactions avec le complexe militaro-industriel. Si une telle architecture économique de l'URSS date d'abord de la collectivisation stalinienne et de la mobilisation totale des ressources du pays pour défendre la «forteresse du socialisme assiégé», le développement d'un archipel scientifique date, lui, des années 1950, lorsque la course aux armements et aux technologies engagées entre l'Union soviétique et les États-Unis a demandé une mobilisation totale non plus seulement des ressources naturelles et industrielles, mais également intellectuelles. Des dizaines de villes scientifiques sont alors créées pour accueillir centres de recherches ou bureaux de conception organisés dans un système dont le but était d'affirmer la supériorité technologique et militaire de l'Union soviétique. La répartition territoriale de ces villes répond d'ailleurs à cet impératif : certaines d'entre elles (ce sont surtout les villes du complexe nucléaire de l'Oural) n'avaient aucune existence officielle, n'apparaissaient sur aucune carte et demeuraient fermées à qui n'avait pas de laisser-passer. D'autres, en périphérie des grandes agglomérations (Leningrad, Moscou et Novossibirsk surtout) pouvaient ne faire l'objet que de très faibles restrictions d'accès, surtout investies dans la recherche fondamentale ou l'innovation civile (Akademgorodok 
ANALYSE GÉOPOLITIQUE DES ENJEUX D’UNE POLITIQUE DE PUISSANCE

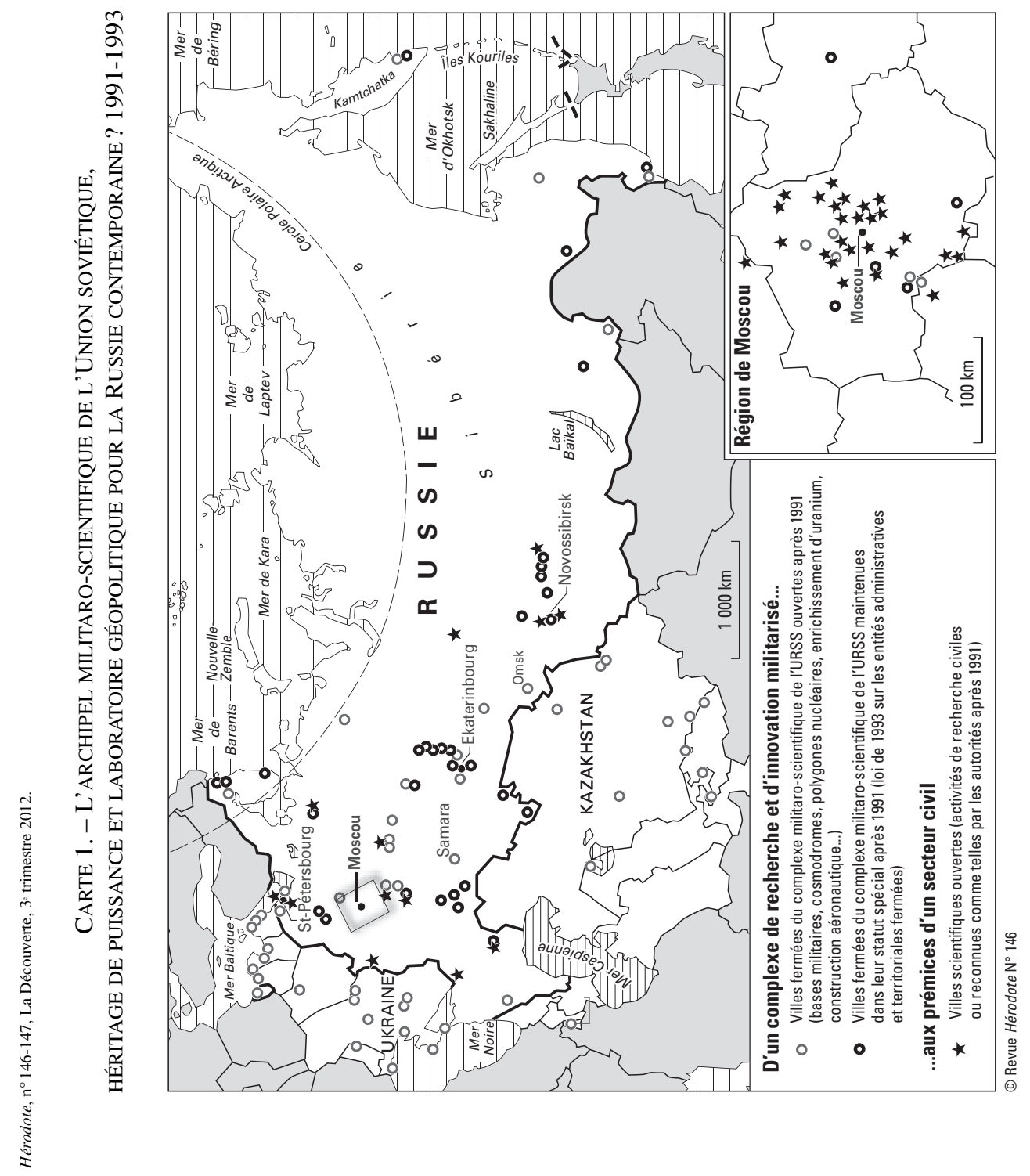




\section{HÉRODOTE}

de Novossibirsk, ou encore Doubna). Leur localisation en périphérie éloignée des grands centres urbains répondait à plusieurs raisons allant de la sécurité (en cas d'attaque nucléaire, les centres scientifiques et leurs précieuses populations seraient épargnés) au bien-être des habitants.

La question du bien-être permet d'aborder le deuxième point concernant des identités urbaines particulières. En effet, on vivait dans ces villes selon des standards supérieurs à la moyenne soviétique. Les magasins y étaient mieux approvisionnés, les infrastructures (urbaines, socioculturelles) mieux entretenues et plus nombreuses. La raison de ces privilèges est à trouver dans le fait que ces villes assuraient des fonctions vitales au maintien et au développement de la puissance soviétique. Les tâches qu'elles remplissaient dans le plan étant souvent d'une priorité absolue, ces villes ont donc été pensées, dans leur urbanisme comme dans leur population, comme des villes d'élite au service des plus hauts intérêts de l'État. La concentration de ces populations scientifiques dans des villes privilégiées a amené à l'émergence de véritables microclimats socioculturels qui constituent aujourd'hui encore une part très importante de l'identité de ces villes.

Troisièmement, ces villes ont été façonnées par l'aspiration soviétique à la puissance, et en constituent aujourd'hui encore le patrimoine le plus important. Or il convient de remarquer ici que la puissance soviétique était une puissance idéologique, en cela que la recherche d'une supériorité absolue sur les Etats-Unis puisait sa légitimité dans la défense d'un modèle à visée utopiste reposant sur une lecture scientiste du monde et de ses rapports de forces. Si le programme spatial ou nucléaire de l'URSS constituait en effet le symbole d'un hard power soviétique, il convient de garder à l'esprit que, dans la conception marxiste-léniniste, ils étaient également pensés comme des processus dialectiques inhérents à la construction d'une société nouvelle, et dont la dimension utopiste résidait dans la maîtrise totale de l'univers par la science. Les exploits de l'URSS dans le domaine scientifique et technique sont donc entrés dans le récit identitaire soviétique avec d'autant plus de force que celui-ci était façonné par une lecture idéologique du destin national.

Dans ce cadre, la fonctionnalité du territoire soviétique, alliée à l'importance stratégique et idéologique du complexe scientifique, a abouti à la formation d'un véritable «archipel de la puissance» soviétique identifié par ses habitants comme l'«avant-garde du communisme».

La chute de l'URSS a induit dans ces villes non seulement une baisse drastique des budgets, mais également une perte de sens quant au rôle de ces installations. En disparaissant, le marxisme-léninisme a de fait emporté avec lui la sacralisation du complexe scientifique et a plongé l'archipel scientifique dans une crise économique et morale sans précédent. De plus, la fin de l'orgnabor («répartition fonctionnelle des forces laborieuses du socialisme») et du système de la propiska (permis de séjour) rendait les gens libres de s'installer où ils le désiraient. 
La conjugaison de ces grands facteurs a initié une importante fuite des cerveaux vers l'étranger ou les grandes villes, ainsi qu'une multiplication des démissions de chercheurs et ingénieurs qui, n'étant plus payés, ou plus aussi bien, se sont tournés vers une économie de subsistance (PME). Malgré quelques actes hérö̈ques et l'opiniâtreté de certains chercheurs pour maintenir leurs outils de travail en état de marche [Coudrec, Franceschi, 2003], la situation a rapidement débouché sur une perte des savoir-faire et la destruction de certaines infrastructures de pointe uniques au monde. En outre, la chute des budgets a également touché toute l'infrastructure sociale, culturelle et urbaine de ces territoires. Les ministères de branche et leurs instituts au sein des villes ne pouvaient par exemple plus payer l'entretien d'un palais de la culture ou d'un gymnase, qui leur appartenait souvent. Ainsi, au début des années 1990, non seulement le complexe scientifique était directement menacé dans son excellence, mais également dans la préservation de microclimats privilégiés que certains décideurs comparaient alors à ceux des cities of knowledge américaines - ces centres périurbains de haute technologie et de recherche qui avaient su, dès les années 1950, attirer les cerveaux notamment grâce à un cadre de vie agréable [Pugh O'Mara, 2004], jusqu'à devenir plus tard des territoires mondialement renommés (Silicon Valley par exemple).

Des stratégies de survie aux stratégies de renouveau: le statut de naukograd ou la systématisation du concept de sauvegarde patrimoniale

À partir de 1992, c'est donc tout un ensemble hétéroclite de villes d'élite qui sont confrontées au même problème, celui de leur survie. En effet, pour celles situées en Sibérie par exemple, l'éloignement et l'isolement ne sont plus compensés par l'assurance d'un cadre de vie supérieur. Pour les villes scientifiques situées en périphérie des grandes agglomérations russes, la force d'attraction des métropoles, décuplée par la fin du système de propiska, constituait un danger considérable. Par exemple, des villes d'élite de la banlieue de Moscou, les plus importantes sont trop éloignées de la capitale (ou mal desservies) pour devenir des cités-dortoirs, mais demeurent dans une zone d'attraction qui risque de drainer tout le potentiel intellectuel et scientifique sur lequel repose le potentiel économique de ces municipalités.

Face à ce dilemme, les administrations de ces villes mirent rapidement en place des groupes de réflexion composés des décideurs politiques et économiques au niveau municipal. Ces différentes villes ont alors commencé à organiser des conférences et tables rondes intermunicipales sur l'avenir de leur patrimoine scientifique, intellectuel et infrastructurel ainsi que sur les moyens de le valoriser. Si ces différentes villes n'entretenaient pas à l'époque soviétique de relations 


\section{HÉRODOTE}

administratives directes (elles dépendaient de ministères souvent différents), la collusion des réseaux de l'Académie des sciences et des divers instituts de recherche favorisa un rapprochement rapide. Dès la fin de l'année 1992, à l'issue d'une conférence tenue à Obninsk apparaît ainsi le terme de Naukograd (cité des sciences) pour définir un nouveau type de villes qui partageraient de nombreuses caractéristiques communes (population hautement qualifiée, nombreuses infrastructures issues de l'époque soviétique, laboratoires de recherche, cadre de vie supérieur...). Ces villes fondent l'année suivante une Union pour le développement des Naukograds, dont le but est, une fois définies leurs particularités communes, de faire porter au niveau fédéral la voix d'une nouvelle démarche de développement de la science et de l'innovation fondée sur la mise en valeur de structures héritées de l'époque soviétique.

Il convient de noter ici que la faiblesse structurelle du pouvoir central russe tout au long des années 1990 a permis à une telle initiative de voir le jour; la mise en valeur du potentiel de ces villes a ainsi été strictement portée par les pouvoirs municipaux et régionaux, sans l'intervention d'un pouvoir fédéral qui laissait alors se développer des systèmes décentralisés. C'est pourquoi, dès 1993, est engagée une campagne de lobbying au niveau central, afin de faire entrer dans la loi ce terme de Naukograd, forgé par des élites locales. L'idée est alors de créer un statut administratif à part entière reconnaissant les particularités héritées de l'époque soviétique et considérées comme inhérentes à l'identité de ces villes. Malheureusement, la crise constitutionnelle de 1993 vient ruiner ces espoirs, et il faut attendre 1999 pour que soit finalement votée la loi sur les Naukograds de la Fédération de Russie. Cette année-là, deux projets furent proposés. L'un émis par la Douma, et l'autre par le Conseil de la Fédération (Chambre haute). C'est finalement le projet du Conseil qui l'emporte, celui de la Douma ayant été bloqué par un veto du président Eltsine, en cela que le projet refusé comportait un préambule qui définissait les Naukograds au-delà d'une stricte appréciation chiffrée de leur potentiel scientifique. Ainsi, dans la loi votée en 1999, pour obtenir le statut de Naukograd, une ville doit par exemple avoir au moins $50 \%$ de sa population active travaillant dans le secteur des sciences et technologies. Cette définition, strictement numéraire et statistique, ne répondait pas totalement aux attentes de l'Union pour le développement des Naukograds, qui espérait faire entendre au plus haut sommet de l'État les particularités intrinsèques de ces villes par rapport à leur économie, mais aussi par rapport à leur histoire et leur identité. Des critères objectifs ne suffisaient pas, selon 1'Union, pour faire correspondre la loi avec la réalité sociale, culturelle et économique de ces villes. De son côté, le Président a bloqué le projet de la Douma en arguant que cela aurait été un octroi de privilège pour quelques villes que d'accepter des critères subjectifs dans l'évaluation. 
Cette affaire montre ainsi que la représentation des Naukograds n'est pas la même au niveau municipal et au niveau fédéral. En fait, bien que l'Union ait accusé le pouvoir fédéral d'être déconnecté des réalités socioéconomiques de la province, la décision du président de la Fédération suit une autre logique: en définissant subjectivement ces villes, l'Union serait parvenue à ses fins en faisant graver dans le marbre une idée, une représentation bien plus qu'une réalité économique. Or, en acceptant cela, le pouvoir central s'aliénait une conception des centres scientifiques formulée par une organisation composée par des pouvoirs locaux et régionaux agissant d'abord dans leur intérêt, à travers une mise en scène particulière du patrimoine soviétique.

Les Naukograds, ou l'invention d'une «idéologie de la science » sur fond de réseaux clientélistes

Car si certains ténors du programme des Naukograds n'hésitent pas à proclamer que ces villes constituent une véritable «idéologie de la science et de l'innovation ${ }^{6} »$, il convient de rappeler qu'elles sont d'abord le résultat d'une entente politique locale, entre plusieurs réseaux qui trouvèrent leur intérêt à promouvoir l'idée d'une destinée commune. Le concept de Naukograd, légitimé par une idée de sauvegarde et de renaissance du patrimoine scientifique, demeure un faire-valoir pour des élites locales en quête perpétuelle de légitimité politique et de ressources financières «grises» permettant de maintenir une cohésion dans les réseaux locaux de pouvoir. L'apparition du terme de Naukograd en 1993 résulte d'abord d'une négociation de différents pouvoirs municipaux qui disposaient de réseaux communs au sein de l'Académie des sciences, des ministères de branche ou du Parti. D'ailleurs, la carte des différentes villes membres de l'Union des Naukograds prouve cette proximité de diverses élites en cela que l'initiative fut au départ concentrée dans la région de Moscou. Ces villes partagent des réseaux d'influence communs via le gouvernement de la région de Moscou, qui fut d'une aide salvatrice dans la politique de lobbying menée par l'Union.

L'existence d'un potentiel intellectuel et d'infrastructures sociales et économiques uniques, sur lequel se fonde l'idée de Naukograd comme représentation, procédait en fait de politiques sectorielles qui n'entretenaient avant 1991 aucun lien entre elles, à cause du cloisonnement vertical du système soviétique de recherche et d'innovation. L'invention du terme de Naukograd s'apparente donc à

6. Selon les mots de M. Safariliev, ancien président du comité parlementaire de la Douma sur les villes de science et les politiques de décentralisation. 
HÉRODOTE

CARTE 2. - LA LENTE ÉMERGENCE DES TERRITOIRES DE SCIENCE ET D'INNOVATION DANS LA RUSSIE POSTSOVIÉTIQUE: DU COMPLEXE MILITARO-INDUSTRIEL

À L'ÉCONOMIE DE MARCHÉ, UNE INITIATIVE DES POUVOIRS LOCAUX 1993 -2001

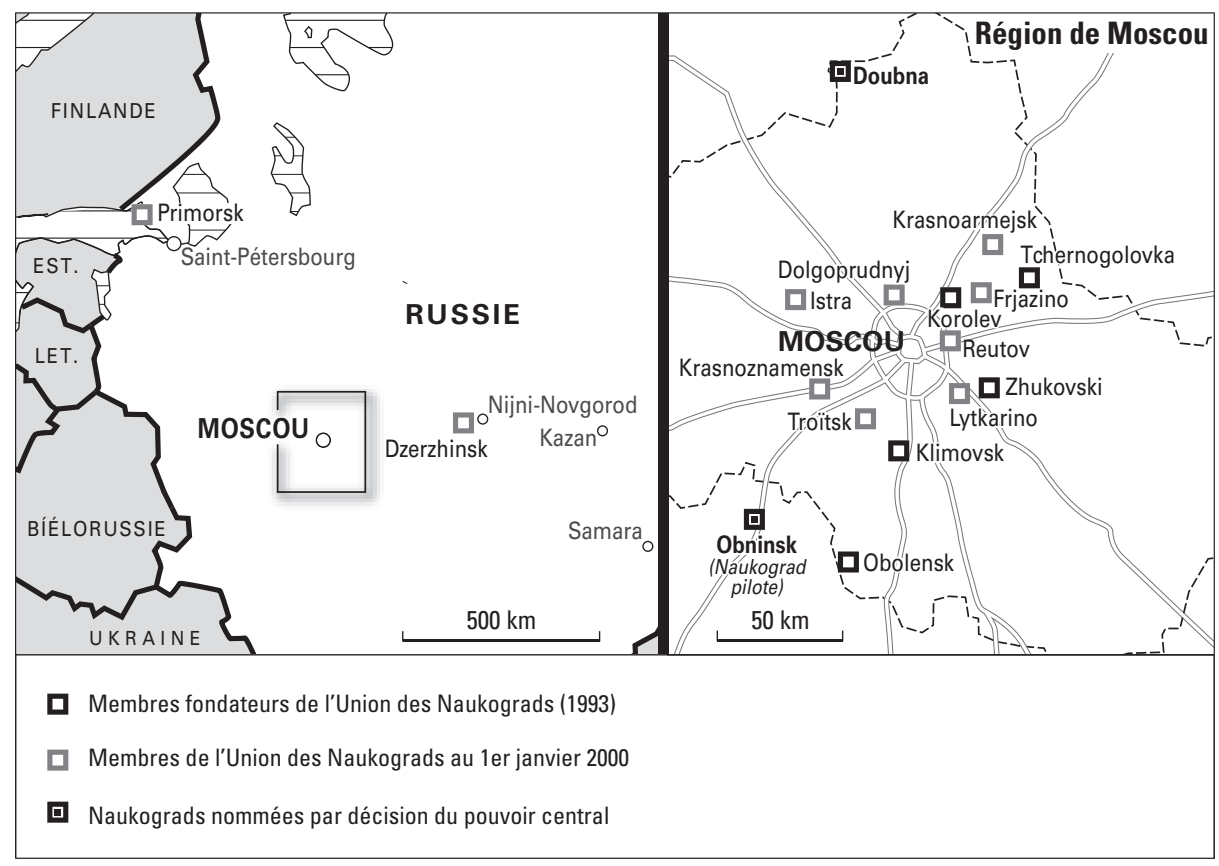

(c) Revue Hérodote $N^{\circ} 146$

une véritable «mise en scène» d'un patrimoine désigné comme commun, mais qui en réalité dépendait profondément des politiques sectorielles des ministères soviétiques. Certaines villes, à l'image de Doubna, étaient tournées vers le nucléaire et comportaient d'importants centres de recherche fondamentale où l'on s'installait durablement après avoir soutenu sa thèse de physique. Au contraire, dans une ville comme Korolev, qui abrite le centre de contrôle des vols spatiaux, on était ingénieur, et l'on ne restait souvent que quelques années avant d'être muté dans une autre ville du complexe aérospatial. Dans ces deux cas, les cultures urbaines de la science étaient bien différentes, bien que l'idée de sauvegarde du patrimoine scientifique, du potentiel intellectuel et des infrastructures ait pu devenir un souci commun pour toutes ces villes ayant subi de plein fouet l'effondrement du système soviétique. 
Ainsi, la mise en place des premiers statuts de Naukograd dès 2000 concerne principalement la rénovation de l'espace urbain avec, curieusement, très peu de dispositifs de soutien aux entreprises innovantes et aux centres de recherche. Dans la période 2000-2006, les villes ayant obtenu le statut administratif de cité des sciences ont vu affluer des milliards de roubles dans leurs caisses afin de sauvegarder et rénover des infrastructures socioculturelles, ou en développer de nouvelles (réseau Internet par exemple). Les entreprises innovantes nées du processus de spin-off des années 1990 se sont retrouvées exclues du programme, et l'attribution d'un soutien financier ou matériel dépendait le plus souvent des relations personnelles que les chefs d'entreprise entretenaient avec les élites municipales. Â Doubna, un plan d'aménagement urbain très ambitieux a ainsi été mis au point, sa réalisation rendue possible par d'énormes subventions versées dans le cadre du programme de Naukograds.

Dans cette ville, ces politiques de grands travaux ont été accueillies très favorablement par une population marquée par la décrépitude des années 1990 et la joie de voir à nouveau la ville fleurir. Cette mise en scène d'un renouveau des structures soviétiques a également favorisé le développement d'un système clientéliste à grande échelle au sein des élites municipales. Au pouvoir depuis la chute de l'URSS et issue des cadres locaux du Parti communiste d'Union soviétique, l'équipe municipale a distribué, par le biais de marchés publics truqués, les dividendes de l'aide fédérale en faisant gonfler (parfois de plus de $200 \%$ ) le coût des contrats publics destinés à la rénovation urbaine et à la sauvegarde d'un environnement innovant.

Dans ce cadre, Doubna est devenue une très belle ville, aux atours presque opulents en comparaison des paysages urbains sinistrés environnants. Or cette prospérité dissimule un modèle de développement économique qui demeure en grande partie tributaire des commandes d'Etat. Les grandes entreprises de la ville issues de l'époque soviétique, ainsi que leur sous-traitance souvent issue des spinoff des années 1990, continuent de travailler essentiellement pour des commandes publiques $^{7}$ à quelques notables exceptions près.

Le poids de ces grandes entreprises se mesure à leur chiffre d'affaires, mais également à un réseau complexe d'allégeances par rapport à des sous-traitants souvent issus de ces mêmes entreprises. Dans les années 1990, le processus de spin-off a bénéficié d'un soutien logistique (mise à disposition de locaux,

7. Les grandes unités de production de Doubna sont toutes héritées de la période soviétique, lorsque la ville était un important centre de recherches fondamentales et de haute technologie: Radouga (avionique et réacteurs pour l'armée de l'air), Specmash («constructions spéciales » du ministère de la Défense), ou Tenzor (équipements de sécurité pour les centrales nucléaires, donc prestataire de l'agence fédérale ROSATOM qui gère les centrales). 
HÉRODOTE

CARTE 3. - LA CITÉ DES SCIENCES DE DOUBNA EN 2012.

DÉFIS POSTSOVIÉTIQUES D'UNE RECOMPOSITION TERRITORIALE ET ÉCONOMIQUE

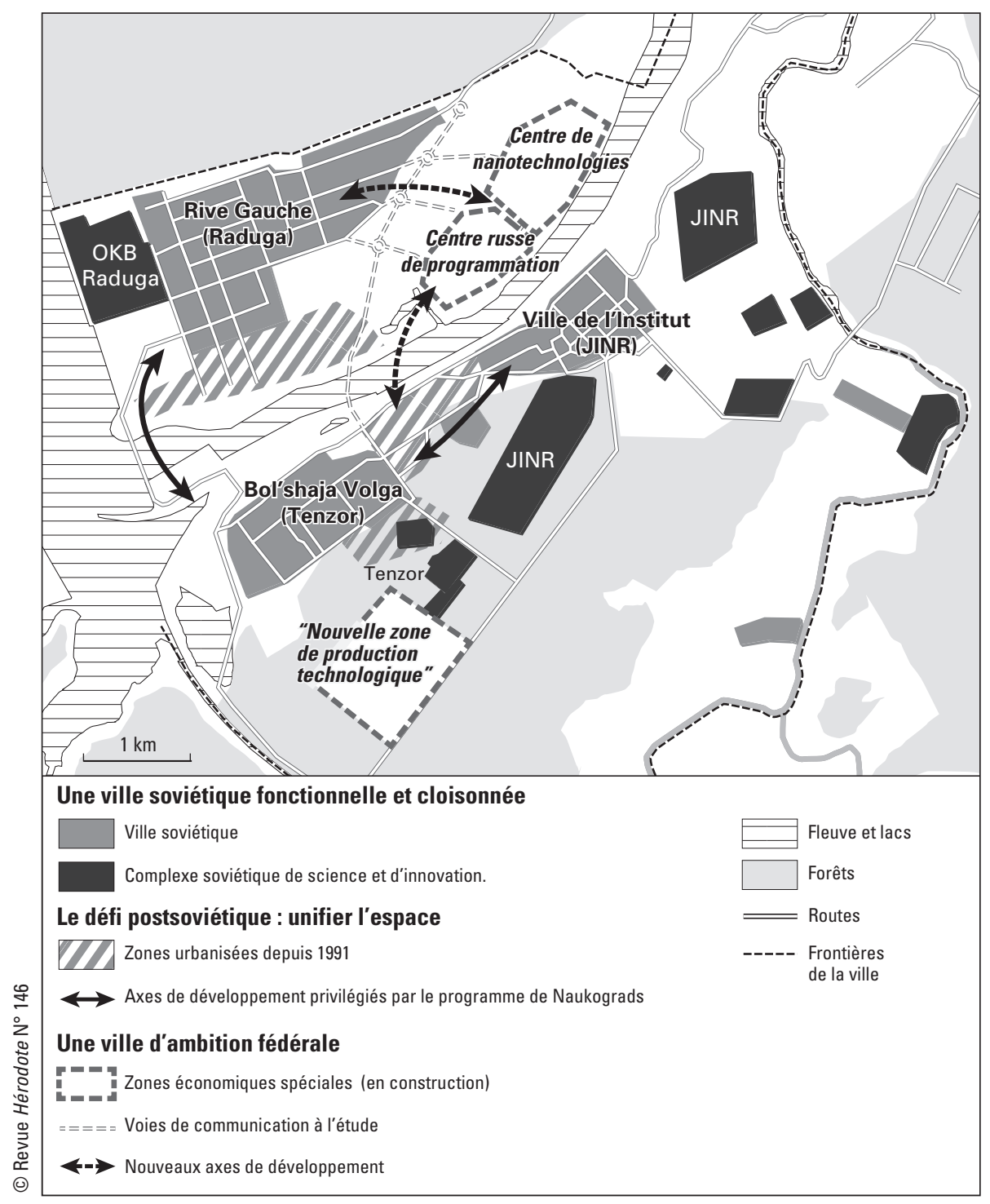

208 
de matériel) de la part de ces même grandes entreprises qui, voyant leur activité diminuer de manière significative, ont bradé une partie de leur patrimoine en échange d'allégeances clientélistes. La connivence historique des autorités municipales avec la direction des grandes entreprises s'est superposée à ce système, renforcée par les importantes ressources budgétaires allouées par le niveau fédéral.

Les Naukograds constituent donc aujourd'hui un système de recherche et d'innovation fondé sur la mise en valeur du patrimoine soviétique, mais soumis à de fortes pressions clientélistes qui en ralentissent le développement économique. Une entreprise ne bénéficiant pas de soutien de la part des élites aura ainsi beaucoup de mal à s'implanter.

Des rivalités de pouvoir à l'émergence d'une contestation citoyenne,

le renouveau comme objet de discorde

Le rapport que les autorités centrales entretiennent avec ces systèmes locaux est complexe. D'une part, la réussite de certains plans de réaménagement (comme à Doubna) fournit de véritables vitrines au renouveau de la science et de l'innovation depuis le début des années 2000. D'autre part, les détournements et systèmes locaux d'allégeance s'insèrent dans la logique globale du système politique russe qui consiste à acheter le soutien des élites locales.

Cependant, abandonné par le niveau fédéral durant toute la décennie 1990, le secteur de la science et de l'innovation redevient une préoccupation nationale à partir de 2002. Cette année-là, une loi sur la fondation de technopôles en Sibérie marque le retour de l'État central dans la gestion territoriale du complexe scientifique avec quelques premières timides initiatives de création de nouveaux milieux innovants. Cette première impulsion est suivie en 2005 par la création des premières Zones économiques spéciales (ZES) de haute technologie, au sein desquelles les entreprises partenaires bénéficient d'exonérations fiscales et de soutien à l'exportation. Parfois installées dans des Naukograds, ces ZES signent le retour effectif du pouvoir central dans les décisions locales concernant le secteur des hautes technologies. La composition des conseils d'administration de ces ZES a en effet pu être un savant dosage entre élites locales et représentants fédéraux, initiant un début de cohésion entre les deux niveaux tout en maintenant les réseaux clientélistes au niveau municipal.

Mais avec l'annonce de la création de Skolkovo en 2009, c'est une tout autre logique qui est mise en avant. À grand renfort de communication médiatique, l'ambition du président Dmitri Medvedev a été ici de créer ex nihilo un nouveau «milieu innovant» capable d'attirer des investisseurs de tout premier plan. 


\section{HÉRODOTE}

L'invention du terme innograd (cité d'innovation) pour définir Skolkovo s'oppose ainsi à celui de naukograd pour légitimer un processus économique et politique différent. Par cette distinction, le pouvoir fédéral entend créer une séparation claire entre la mission des uns et des autres. Les Naukograds seraient des incubateurs de recherche et d'innovation à ambition régionale, alors que Skolkovo serait une véritable fenêtre sur le monde, un laboratoire à idées destiné à essaimer en Russie et au-delà (Innopolis Kazan, projet de «Skolkovo militaire», ou la création d'une filiale de Skolkovo en Biélorussie). Cette dichotomie est cependant critiquée par l'Union des Naukograds en cela que les énormes financements dont bénéficie aujourd'hui Skolkovo ont en grande partie été ponctionnés sur ceux dévolus aux villes de science. Initiative présidentielle par excellence, Skolkovo est aujourd'hui sourdement critiquée par les réseaux municipaux qui, une fois de plus, mettent en avant leur patrimoine et leur «facteur humain » pour se distinguer d'un projet central extrêmement coûteux et dont les résultats ne seraient pas à la hauteur des attentes. Contrairement à Skolkovo, les Naukograds disposeraient selon eux de tout le potentiel humain nécessaire à leur transformation en centres de recherche et d'innovation de niveau mondial. Leur argument phare consiste à dire qu'une Silicon Valley russe doit s'inspirer du modèle californien: cadre de vie agréable, liberté des investisseurs... Des atouts déjà présents dans les villes de science, alors que Skolkovo demeure un simple chantier aux abords du MKAD (le périphérique de Moscou), dont le choix du lieu aurait été dicté par d'obscures affaires de spéculation immobilière ${ }^{8}$.

Or, à cette rivalité entre pouvoirs locaux et Kremlin, se superpose celle, plus méconnue dans ses rouages, entre Poutine et Medvedev. Dans la presse, Skolkovo est allègrement présenté comme l'enfant de Medvedev et de son obsession pour la modernisation, tant moquée par les opposants. Vladimir Poutine avait quant à lui soutenu la stratégie des Naukograds, notamment en déclarant à Doubna, pendant la dernière campagne présidentielle vouloir, y promouvoir un «centre de recherche mondialement reconnu». Vladimir Poutine a d'ailleurs créé en mai 2010 une Agence pour les initiatives stratégiques (ASI) destinée à soutenir les initiatives innovantes de PME de province. À l'époque, l'ASI avait pu être vue comme la réponse de Poutine au projet de Skolkovo, auquel il serait plutôt opposé. Toutefois, le rôle de l'ASI demeure aujourd'hui peu clair. Si son action est fondée sur des réseaux municipaux dans toute la Russie pour soutenir des projets de jeunes

8. Non loin du terrain de Skolkovo se trouve en effet l'ancienne datcha de Leonid Brejnev qui fut, après la chute de l'URSS, récupérée par la mairie de Moscou comme résidence d'élite, autour de laquelle des personnages influents firent construire leur propre résidence secondaire. Certains accusent aujourd'hui ces personnes de vouloir vendre leurs terrains au prix fort aux entreprises qui s'installeront à Skolkovo. 
talents, on a pu l'accuser de faire le jeu du clientélisme municipal. En effet, en tant que président de l'ASI, c'est Vladimir Poutine lui-même qui décide des projets à financer. L'ASI pourrait alors constituer, en même temps qu'une tentative de contrôle sur les réseaux municipaux qui font remonter les projets, une «pépinière» de futurs obligés.

La situation actuelle oppose ainsi plusieurs logiques de développement scientifique qui toutes se réclament du renouveau d'un secteur patrimonial et prestigieux. On l'a vu, ces oppositions puisent leurs racines dans des rivalités entre différents niveaux de pouvoir qui, chacun, promeuvent une logique de «mise en scène» compatible avec leurs intérêts. D'ailleurs, les récentes déclarations du patron de Rosnanotech Anatoli Tchoubaïs (investi dans le projet Skolkovo comme dans la version «Medvedev» de la modernisation russe) sur la fin du «pacte de stabilité» unissant Vladimir Poutine et son pays pourraient indiquer le début d'une nouvelle rivalité ouverte entre le Président et certains oligarques proches de Dmitri Medvedev et de son programme de modernisation dans un savant dosage de déclarations visant à discréditer l'action du Kremlin.

L'opposition d'un système «soviétisant» dans la forme des structures qu'il promeut comme dans la composition de ses réseaux de pouvoir s'oppose alors au modernisme prôné par Dmitri Medvedev durant son mandat présidentiel. Le modèle du jeune milliardaire californien décontracté et intégré à la mondialisation numérique aujourd'hui mis en avant par l'école de management de Skolkovo pour former les futurs cadres du complexe russe de hautes technologies se heurte ainsi à des réalités régionales qui restent ancrées dans la valorisation des acquis soviétiques avec, par exemple, l'entretien d'un camp de pionniers, d'un palais de la culture ou d'un musée à la gloire des grands inventeurs du siècle passé. De même, les méthodes d'administration de la recherche et de l'innovation sont radicalement différentes car elles convoquent deux réalités sociales et culturelles totalement déconnectées. De cette dichotomie née lors des années Medvedev, demeure la question de son évolution sous Vladimir Poutine, qui n'a jamais caché sa désapprobation pour les Mark Zuckerberg russes en puissance.

\section{Émergence d'une contestation politique sociale face aux discours de renouveau:} le cas de Doubna

Entre ces deux logiques portées par des réseaux de pouvoir, il est nécessaire de distinguer la position du citoyen et les critiques susceptibles d'émerger par rapport à l'une ou l'autre des mises en scène. Pour Skolkovo, on l'a vu, le projet n'étant encore qu'au stade expérimental, la contestation se borne à la sphère des réseaux sociaux et des déclarations de l'opposition qui dénonce une mascarade. 
HÉRODOTE

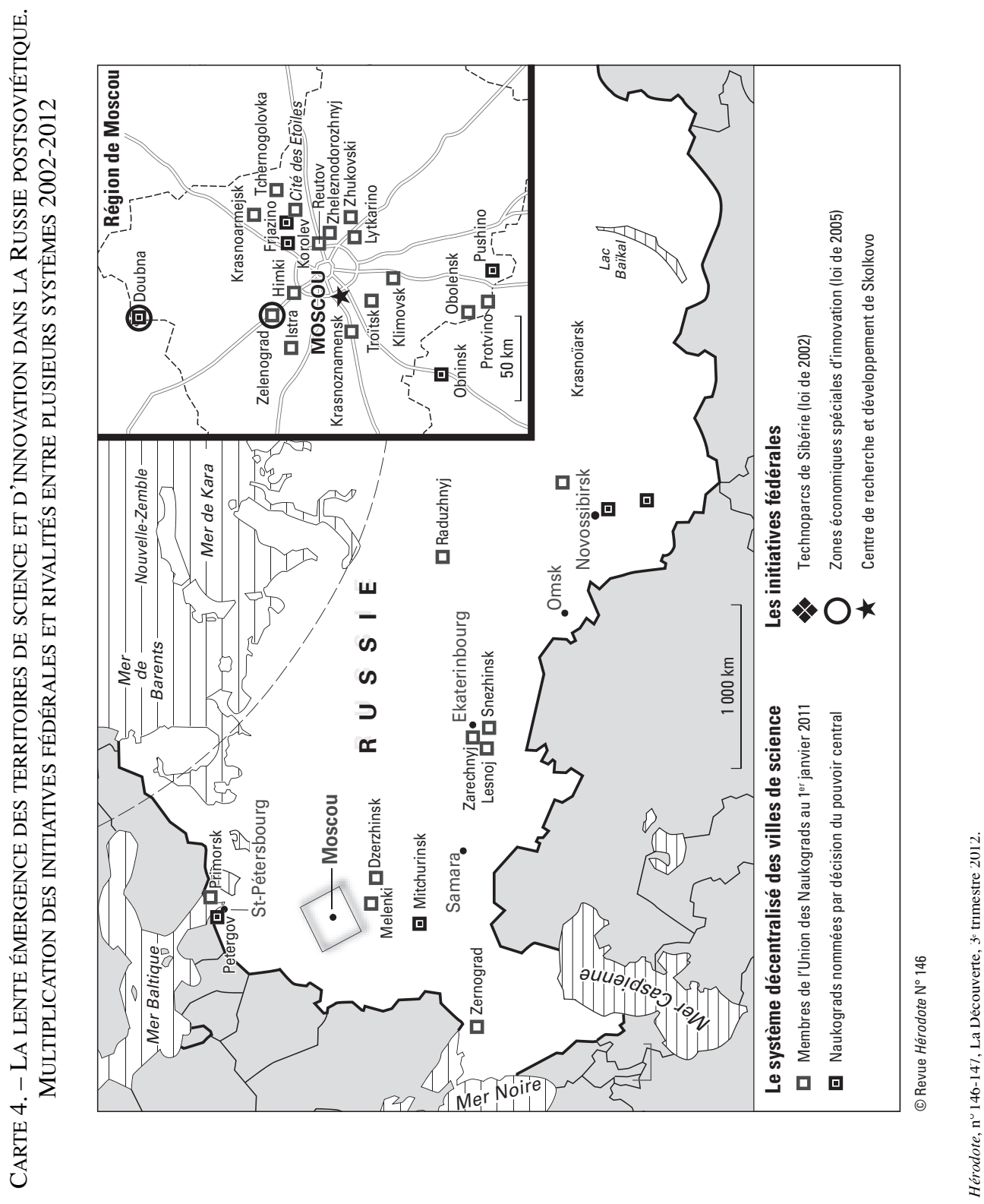

212 
Dans les Naukograds, en revanche, existe un vieux terreau contestataire dont l'origine remonte à l'époque soviétique et qui, aujourd'hui, subit de profondes mutations évocatrices de possibles évolutions politiques par rapport aux discours du renouveau.

Dans le cas de Doubna, la faiblesse structurelle du Parti communiste à l'échelon local avant $1991^{9}$ avait induit une tradition de dissidence qui puisait ses discours dans la cohésion internationale de la communauté scientifique. De même, au niveau local, les élites scientifiques entraient souvent en conflit avec les autorités sur des questions d'aménagement du territoire, les physiciens et académiciens au carnet d'adresses bien fourni demeurant très attachés à Doubna en tant que ville d'exception, à la fois proche de Moscou et isolée dans la forêt ${ }^{10}$. Dès le milieu des années 1970, la question de la protection de l'environnement est donc devenue centrale dans la structuration de cette opposition d'élite contre la folie des grandeurs de certains décideurs.

La victoire aux élections municipales libres de 1990 d'une faction dite «opportuniste» du Parti a fait entrer l'opposition dans un jeu politique libéré des contraintes imposées par le socialisme. La préservation de l'environnement unique de Doubna a entraîné les opposants à contrer de nombreuses initiatives qui auraient défiguré la ville à l'initiative d'une équipe municipale en quête d'une stratégie de renouveau.

Si les grands chantiers mis en place dans le cadre du programme de Naukograds ont quelque peu calmé ces vieilles rivalités, la réussite de nombreux

9. À cause, notamment, du fait que l'institut de recherche nucléaire qu'hébergeait la ville était une organisation internationale au sein de laquelle le contrôle politique ne pouvait s'exercer comme dans n'importe quelle structure soviétique du fait des divergences d'approches du socialisme qui pouvaient exister entre les États membres

10. Au milieu des années 1970, le fils de Leonid Brejnev lui-même s'est heurté à cette opposition. Celui-ci voulait faire construire à Doubna une base de repos pour ses collègues hauts fonctionnaires du ministère du Commerce extérieur. Viktor Okhrimenko, ancien maire de Doubna et ingénieur en communications spatiales, raconte comment il a empêché l'affaire d'aboutir dans un savoureux dialogue entre lui-même et les obligés de Yori Brejnev. La scène se passe dans le bureau de l'architecte en chef de la région de Moscou: "Il est dommage que nous n'ayons pu trouver un accord avec vous. Yuri Leonidovitch ne sera pas content", me dirent [les représentants du Commerce extérieur]. "Excusez-moi mais qui est ce Yuri Leonidovitch?" demandai-je. "C'est le vice-ministre au Commerce extérieur, Yuri Leonidovitch Brejnev." Là j'ai vu rouge: "C'est du chantage! Comment osez-vous utiliser le nom du premier-secrétaire! Je parlerai personnellement de vous à Leonid Ilitch (Brejnev)." Alors l'architecte en chef, qui assistait à cet échange musclé, racla sa gorge et se tourna vers les types du Commerce extérieur: "Camarades, s'il vous plaît, stop! Je vais vous trouver un bien meilleur endroit pour vos activités, et plus près de Moscou!"» Témoignage recueilli par Nikolai Prislonov. 


\section{HÉRODOTE}

projets de modernisation a attiré une nouvelle génération d'habitants. En recherche d'un lieu agréable pour fonder une famille tout en étant proche de la capitale, c'est une jeunesse qualifiée qui est désormais attirée par Doubna, modifiant profondément sa composition culturelle et politique. Les méthodes clientélistes de gestion de l'administration municipale ont rapidement constitué un terreau d'indignation pour ces jeunes nés dans les années 1980, voyageurs, aspirant à monter leur entreprise et à s'investir dans le monde associatif local. Comme leurs aînés scientifiques, ces jeunes opposants sont révoltés par le «copinage» et l'obscurité totale qui entourent les décisions municipales. Ces deux générations, qui incarnent deux mondes différents mais liés par leur investissement dans le secteur de la science et de l'innovation, se sont retrouvées autour du thème de la défense de l'environnement exceptionnel de Doubna comme sur la nécessité de mener des politiques de renouveau transparentes et honnêtes.

Privées de la manne financière fédérale des années 2000-2006, les autorités municipales ont en effet dû développer de nouvelles stratégies pour maintenir la cohésion des divers réseaux de pouvoir et de clientélisme sur la base de services rendus. Mais l'existence d'une population traditionnellement dissidente et désormais nourrie d'un sang jeune qui aspire à l'honnêteté politique rend la situation de plus en plus délicate. Récemment, la municipalité a ainsi dû léguer des terrains au gouvernement de la région de Moscou, en échange d'autres parcelles nécessaires à la construction de la Zone économique spéciale voulue par les autorités fédérales. Or les terrains donnés par la municipalité aux termes d'un arrangement obscur figurent parmi les lieux emblématiques de l'identité de Doubna. Un lac assez propre pour se baigner, une forêt... De nombreux hommes d'affaires seront sans doute intéressés pour y construire des infrastructures touristiques: les endroits non pollués et agréables à distance raisonnable de Moscou ne sont pas nombreux. Mais en touchant au patrimoine écologique de la ville, la municipalité s'est attirée l'hostilité de tous les mouvements d'opposition. La défense de l'administration, si elle consiste à dire que le renouveau de la ville passe par quelques sacrifices et que la nouvelle Zone économique spéciale garantira une qualité de vie optimale, ne convainc pas grand monde. Le fait que le maire ait pris seul cette décision s'est superposé à un sentiment d'exaspération qui se nourrit du contexte général, menant à une cuisante défaite de Russie unie à Doubna lors des dernières législatives.

Tout en renouant avec la tradition soviétique d'une dissidence d'élite et de résistance face aux promoteurs moscovites, l'opposition se structure et propose aujourd'hui une vision alternative du renouveau de Doubna fondée sur des idées similaires à celle avancée par le pouvoir en place: défense de la qualité de vie comme socle de l'identité municipale, promotion d'un milieu innovant... La différence entre les uns et les autres se situe aujourd'hui sur le terrain de l' «honnêteté», la corruption et le «copinage». Après une période de croissance importante mais 
artificiellement stimulée par les autorités fédérales, la situation actuelle de Doubna est symptomatique d'une Russie qui croit de moins en moins aux rhétoriques du renouveau et à une mise en scène qui s'essouffle. La situation locale à Doubna est alors comparable à celle que l'on retrouve autour des polémiques de Skolkovo. La corruption généralisée, si elle a pu jusque-là être maquillée par l'argent des hydrocarbures, se révèle à l'aune d'une crise économique qui n'épargne pas la Russie, dont les perspectives de croissance ont été revues à la baisse du fait de l'évolution des cours du gaz.

\section{Conclusion}

L'un des défis majeurs de la Russie actuelle, en cela qu'il touche aux conditions futures de son développement, a donc été l'invention d'une nouvelle identité pour la science et l'innovation à des fins non seulement économiques, mais surtout politiques... Aujourd'hui marqué par une dichotomie profonde entre les réformes entreprises par Dmitri Medvedev (Skolkovo) et une logique plus «traditionnelle», le secteur science et innovation demeure, dans la formulation de politiques cohérentes, tributaire de rivalités induites par différents projets, portés par différents niveaux ou réseaux de pouvoir. Les diverses visions du secteur qui se confrontent aujourd'hui inventent chacune leur légitimité dans un rapport au patrimoine matériel de l'URSS et à sa présente mise en scène. L'incohérence de ces diverses visions du renouveau, motivées par des rivalités politiques et même clientélistes, alimentent ainsi une contestation grandissante du pouvoir, en cela que la science et l'innovation concernent une population jeune et formée qui éprouve aujourd'hui un besoin de transparence en contradiction avec vingt années de développement du secteur. La science et l'innovation constituent ainsi un prisme d'analyse géopolitique qui, en bénéficiant d'une assise territoriale héritée en grande partie de İ l'URSS, permet l'analyse d'enjeux contemporains qui touchent aux questionnements sur la nature de la puissance russe. 
HÉRODOTE

\section{Bibliographie}

BASKaKova Ju. M. (2008) Derjavnost' i racional' nyj vybor vo vneshnej poitike («L'aspiration à la grandeur et le choix rationnel en politique »), Moscou.

BOL'SHAKOV V.I., ERMACKOV E.I. (1999), Russkaja sobornost', obshinost' i derjavnost' : istoriko-sociologicheskij analiz ( «La communauté russe des croyants, le génie social et l'aspiration à la grandeur; analyse sociohistorique »), Institut de recherches sociopolitiques de l'Académie des sciences de Russie, Académie des sciences sociales, éditions de l'Académie des sciences, Moscou.

COUDREC M.-L., FRANCESCHI (2003), «La restructuration d'une ancienne ville fermée en Russie: la création de PME autour de l'Institut Vektor de Koltsovo », Revue région et développement, $\mathrm{n}^{\circ} 18$.

PeTrosian D.I., SVINCOV I.D. (2008), Rossija i sovremennyj Mir : problemy politicheskogo razvitija ( La Russie et le monde contemporain: problèmes du développement politique $»), 4^{\mathrm{e}}$ conférence internationale transdisciplinaire, Moscou.

Pugh O'MARA M. (2004), Cities of knowledge, Cold War Science and the Search for the Next Silicon Valley, Princeton University Press, Princeton. 